\title{
BMI open Protocol for a randomised controlled trial to improve cognitive functioning in older adults: the Iowa Healthy and Active Minds Study
}

\author{
Fredric D Wolinsky, ${ }^{1,2,3}$ Mark W Vander Weg, ${ }^{2,4,5}$ M Bryant Howren, ${ }^{4,5}$ \\ Michael P Jones, ${ }^{6}$ Rene Martin, ${ }^{3,5}$ Tana M Luger, ${ }^{4}$ Kevin Duff, ${ }^{7}$ \\ Christopher Goerdt, ${ }^{2}$ Steven Wolfe, ${ }^{2}$ Megan M Dotson ${ }^{1}$
}

To cite: Wolinsky FD, Vander Weg MW, Howren $\mathrm{MB}$, et al. Protocol for a randomised controlled trial to improve cognitive functioning in older adults: the lowa Healthy and Active Minds Study. BMJ Open 2011;: $:$ e000218.

doi:10.1136/

bmjopen-2011-000218

- Prepublication history for this paper is available online. To view these files please visit the journal online (http:// bmjopen.bmj.com).

Received 14 June 2011 Accepted 22 June 2011

This final article is available for use under the terms of the Creative Commons Attribution Non-Commercial 2.0 Licence; see http://bmjopen.bmj.com

For numbered affiliations see end of article.

Correspondence to Dr Fredric D Wolinsky; fredric-wolinsky@uiowa.edu

\section{ABSTRACT}

Objectives: Gradual age-related cognitive deteriorations are common and are hypothesised to be partially attributable to declines in informationprocessing speed. The lowa Healthy and Active Minds Study will evaluate the efficacy and effectiveness of a computerised visual processing speed training programme (Road Tour, Posit Science Corporation, San Francisco, California).

Methods and analysis: Using a 3:3:4:4 ratio within two age strata (50-64 vs $\geq 65$ years old), 681 men and women attending family care clinics were randomised to four treatment groups: $10 \mathrm{~h}$ of on-site Road Tour training, $10 \mathrm{~h}$ of on-site Road Tour training with $4 \mathrm{~h}$ of booster training at 11 months

postrandomisation, $10 \mathrm{~h}$ of on-site attention control using computerised crossword puzzles (Boatload of Crosswords, Boatload Puzzles, LLC, Yorktown Heights, New York) and $10 \mathrm{~h}$ of at-home Road Tour training using the participant's personal computer. The primary outcome, visual processing speed, was assessed at randomisation and post-training (6-8 weeks postrandomisation), and is being reassessed at 1-year postrandomisation using the Useful Field of View test. Five secondary outcomes (Symbol Digit Modalities Test, Trail Making Tests A and B, Controlled Oral Word Association Test, Digit Vigilance Test, and the Stroop Colour and Word Test) were assessed at randomisation and will be reassessed at 1-year postrandomisation. Seven hypotheses will be tested using intent-to-treat analyses involving multiple linear, logistic, Poisson and negative binomial regression.

Ethics and dissemination: Ethics approval was provided by the University of lowa Institutional Review Board (IRB-03 protocol 200908789). All participants completed signed informed consent prior to enrolment. Road Tour is commercially available from Posit Science Corporation, which provided it to lowa Healthy and Active Minds Study at no cost. All participants will receive a free copy of Road Tour for unlimited perpetual use at study completion.

\section{Clinical Trial Registration}

Number: NCT01165463.

\section{ARTICLE SUMMARY}

Article focus

- Given that age-related declines in cognitive functioning are part of the normal ageing process, there is a pressing need for efficient and effective training interventions that improve cognitive functioning in older adults.

n This protocol paper outlines the design of a study that overcomes several important limitations of a prior, large, multisite randomised controlled trial (RCT) that used memory, reasoning and speed of processing interventions, but found that only the latter effectively translated to improved health outcomes.

- This RCT evaluates the efficacy and effectiveness of a second-generation computerised visual speedof-processing intervention using three modes of delivery (on-site without booster training, on-site with booster training and at-home use) versus an attention control (on-site computerised crossword puzzles without booster training) in improving cognitive processing speed and health outcomes.

Key messages

- This is an RCT protocol.

- lowa Healthy and Active Minds Study is the first RCT to evaluate the efficacy and effectiveness of a commercially available computerised visual speed of processing intervention known as Road Tour.

- If this intervention is successful, the product vendor pledges to make the computerised intervention software available to governments for widespread distribution and use at a fraction of the current commercial cost.

\section{INTRODUCTION}

Some degree of gradual, age-related cognitive decline is recognised as universal and as a normal part of the ageing process. This decline is evident across several domains including memory, orientation, attention, 


\section{ARTICLE SUMMARY}

Strengths and limitations of this study

- This study uses six well-established, objective neuropsychological assessments of cognitive processing speed, as well as three highly reliable and valid self-reported measures of health outcomes in a large sample of men and women 50 years old and older.

- Although the sample is large, it was drawn from just one large primary care centre in which minorities are under-represented and the key assessments are only conducted at randomisation, after initial training (6-8 weeks postrandomisation) and at 1year postrandomisation, thereby reducing the opportunity to demonstrate the long-term effects of the intervention.

abstract thinking and perception. ${ }^{1-4}$ Age-related cognitive changes can best be viewed as one end of a continuum that includes preclinical disease, mild cognitive impairment and dementia. ${ }^{5-8}$

As the brain and the visual system age, many changes occur from the periphery through the central nervous system, contributing to deficits in visual perception and cognition..$^{9-11}$ Deficits are particularly notable in visual tasks requiring high levels of temporal precision (visual speed of processing) and attention (tracking multiple objects). These deficits are significant contributors to declines that emerge in visual cognition and visually guided basic and instrumental activities of daily living (ADLs and IADLs), ${ }^{12} 13$ and can be understood as the consequence of central nervous system changes involving brain plasticity. These brain-plasticity-driven changes are likely important contributors to the speed, memory and cognitive deficits common in normal ageing. ${ }^{14}{ }^{15}$ Brain plasticity, however, also creates an opportunity to strengthen cognitive abilities, and many studies have investigated the effects of interventions targeting specific abilities such as memory, attention control, spatial orientation, inductive reasoning, figural relations and artistic expression, or more globally by targeting multiple domains.

A new generation of these intervention studies is quite promising. Especially encouraging are recent studies that provide '...structured experience in situations demanding executive coordination of skills-such as complex video games, task-switching paradigms and divided attention tasks... ${ }^{14}$ (p. 1). The advantage of these studies is that they 'train strategic control over cognition that does transfer to different environments ${ }^{14}$ (p. 1). Several such studies focus on improving visual processing speed. ${ }^{16-19}$

Salthouse has hypothesised that declines in processing speed adversely affect cognition in two ways - the limited time and simultaneity mechanisms. ${ }^{20}$ Limited time refers to the restriction in the amount of time available to successfully accomplish a task when certain cognitive processes are completed too slowly. Simultaneity operates when slowed information processing promotes the loss of early cognitive processing products through decay or displacement before they are needed for later operations. Extensive evidence supports the speed of processing theory of age-related cognitive decline. ${ }^{19-23}$ Associations between various subjective and objective health-status measures and cognitive functioning are also related to processing speed to a greater degree than to other higher-order cognitive processes, suggesting an important link between speed of processing and health outcomes. $^{24}$ Moreover, Salthouse has shown that processing speed peaks at about age 23, plateaus until age 28 and then declines in a linear fashion throughout the remainder of the life course. ${ }^{25}$

One of the most promising interventions focused on improving visual processing speed was developed by Ball and Roenker. ${ }^{19} 20{ }^{26}$ It improves the efficiency and accuracy of visual information processing and the ability to perform complex visual attention tasks. Specifically, users are trained to improve the speed and accuracy with which they identify and locate visual information using a divided attention format. Over time, the difficulty and complexity of each task are systematically increased as users attain specified performance criteria. Manipulations to increase difficulty include decreasing the duration for which visual stimuli are presented, adding visual or auditory distracters, increasing similarity between target and distracter stimuli, and presenting visual targets over a broader spatial expanse. The basic tasks, however, are always the same-central discrimination and peripheral target location.

Ball and Roenker's ${ }^{19} 20{ }^{26}$ programme was extensively evaluated in the Advanced Cognitive Training for Independent and Vital Elderly (ACTIVE) trial. ${ }^{27}$ ACTIVE hypothesised that each of three intervention arms (memory, reasoning and visual processing speed training) would have a direct effect on targeted, trained outcomes (proximal outcomes) and non-specific effects on its non-targeted, untrained outcomes (via social contact or cognitive engagement mechanisms). The reasoning and memory interventions were expected to affect only everyday problem solving and ADLs and IADLs, whereas the speed of processing intervention was hypothesised to have more diverse effects, including ADL and IADL functioning, everyday speed and driving habits. All three interventions were expected to affect the secondary outcomes, including health-related quality of life (HRQoL), depressive symptoms and locus of control. Although all ACTIVE treatments were effective at improving their targeted abilities, visual speed of processing led to the largest gains. ${ }^{28}{ }^{29}$ Moreover, the greatest relative improvements were clearly associated with visual speed of processing training as well, which produced effect sizes more than double those associated with the two other interventions at every time point.

The effects of ACTIVE's visual speed of processing intervention (vs the no-contact control group) on the secondary or health outcomes have been shown as well. These included: (1) a $\$ 244$ per person-year (3\%) reduction $(p=0.012)$ in predicted medical expenses at 
1 year $^{30} ;(2)$ a $38 \%$ reduction in the risk of global decline in HRQoL at 2 years $(\mathrm{p}=0.004)$ and a $25.6 \%$ reduction in the risk of global decline in HRQoL $(\mathrm{p}=0.038)$ at 5 years ${ }^{31}{ }^{32}$; (3) a $30 \%$ reduction in the risk of worsening depressive symptoms at both 1 year $(\mathrm{p}=0.012)$ and 5 years $(\mathrm{p}=0.023)^{33}$; $(4)$ a $38 \%$ reduction in the risk of the onset of suspected clinical depression at 1 year $(\mathrm{p}<0.01)^{34} ;(5)$ improvements in self-rated health at 2,3 and 5 years equivalent to at least half of the difference between 'excellent' and 'very good' responses $(p<0.05)$, which is known to be associated with a $0.8 \%$ absolute reduction in the 5 -year mortality, and a $10 \%$ relative mortality reduction ${ }^{35}$; and (6) a $64 \%$ greater likelihood $(p<0.05)$ of improvements in internal locus of control at 5 years. ${ }^{36}$ No adverse effects of speed of processing training in ACTIVE have been identified.

As important as it was, however, ACTIVE had five serious limitations. First, ACTIVE used a no-contact rather than an attention control group, making it impossible to rule out placebo effects. Second, ACTIVE's approach to booster training was compliance-conditioned, making it impossible to separate adherence effects from dosing levels. Third, ACTIVE relied on only one speed of processing assessment test (the Useful Field of View, or $\mathrm{UFOV}^{37}$ ), which was sufficiently thematically and procedurally comparable with the speed of processing intervention itself that the results could merely reflect 'training to the test.' Fourth, ACTIVE used an early version of the speed of processing training that required supervised assistance, and is thus not practical for widespread implementation. Finally, ACTIVE included only participants $\geq 65$ and thus cannot be used to address the important issue of whether earlier age-related declines ${ }^{25}$ can be avoided or ameliorated.

\section{CURRENT STUDY}

We designed the Iowa Healthy and Active Minds Study (IHAMS) to overcome ACTIVE's five limitations. IHAMS is a four-group RCT (NCT01165463). The first group received a standard dose $(10 \mathrm{~h})$ of computerised visual processing speed training in our laboratory. The second group also received a standard dose of computerised visual-processing speed training in our laboratory, but they were invited back to the laboratory for $4 \mathrm{~h}$ of subsequent booster training regardless of their adherence to their training. The third group (attention control) received a standard dose of training using computerised crossword puzzles in our laboratory. The final group took the computerised visual processing speed training software home to use it on their personal computer (PC) for at least a standard dose. The primary outcome is visual processing speed, which was assessed at randomisation and after the completion of training (at 6-8 weeks postrandomisation), and will be assessed again at 1-year postrandomisation. Five secondary cognitive processing speed outcomes were assessed at randomisation and will be assessed again at 1 year thereafter.
We specified seven a priori hypotheses $(\mathrm{Hn})$ that we expect to be supported by separate analyses in each age stratum. The first addresses changes in the primary outcome between randomisation and post-testing. Because no standard booster training occurred by this time, and because supplemental training beyond $10 \mathrm{~h}$ in the at-home group should have been minimal, we hypothesise (H1) that participants randomised to Road Tour training (Groups 1, 2 and 4) should have significantly and similarly greater improvements in visual processing speed immediately after training than the attention control group (Group 3).

The six remaining hypotheses address expectations about changes in all six of the primary and secondary outcomes between randomisation and 1-year postrandomisation. H2 replicates ACTIVE (ie, on-site delivery) and hypothesises that the basic and booster effects of visual speed of processing (Group 2) will be significantly greater than those observed for the attention control group (Group 3). To separate the basic effect (Group 1) from the basic plus booster effect (Group 2), we further hypothesise (H3) that Group 1 will also improve significantly more than Group 3 (attention control), but that (H4) Group 2 will improve significantly more than Group 1 . H5 examines the effect of the at-home delivery of the visual speed of processing training (Group 4) versus the effect of the attention control training (Group 3); here we expect a significantly greater improvement for Group 4 than Group 3. $\mathrm{H} 6$ and $\mathrm{H} 7$ evaluate the different modes of implementing the visual speed-of-processing intervention. We hypothesise (H6) that given the potential for individual dosing and maintenance in Group 4 (at-home training) it will have a significantly greater improvement than Group 1 (on-site training without boosters). H7 evaluates the potential for individual dosing and maintenance versus standard booster training; here we hypothesise that the improvements for Group 4 (unlimited at-home dosing) will exceed those for Group 2 (in which standard and booster training doses are fixed).

\section{METHODS AND ANALYSIS}

Figure 1 shows the IHAMS study design and participant recruitment results. IHAMS is a four-arm parallel RCT using a 3:3:4:4 allocation ratio and block randomisation separately within two age strata (50-64 and 65-87 years old). Participants were randomised to one of the following groups: (1) $10 \mathrm{~h}$ (over the first 5-6 weeks) of on-site training using Road Tour (Group 1), (2) $10 \mathrm{~h}$ of onsite training using Road Tour plus $4 \mathrm{~h}$ of booster training at 11 months postrandomisation (Group 2), (3) $10 \mathrm{~h}$ of on-site attention control using computerised crossword puzzles (Group 3) or (4) at-home training using Road Tour for $10 \mathrm{~h}$ or more (Group 4). When enrolment closed in late November 2010, 681 participants had been randomised with 154 in Group 1, 148 in Group 2, 188 in Group 3 and 191 in Group 4. Post-training assessments occurred at $6-8$ weeks postrandomisation, and complete 
Figure 1 IHAMS CONSORT flow diagram

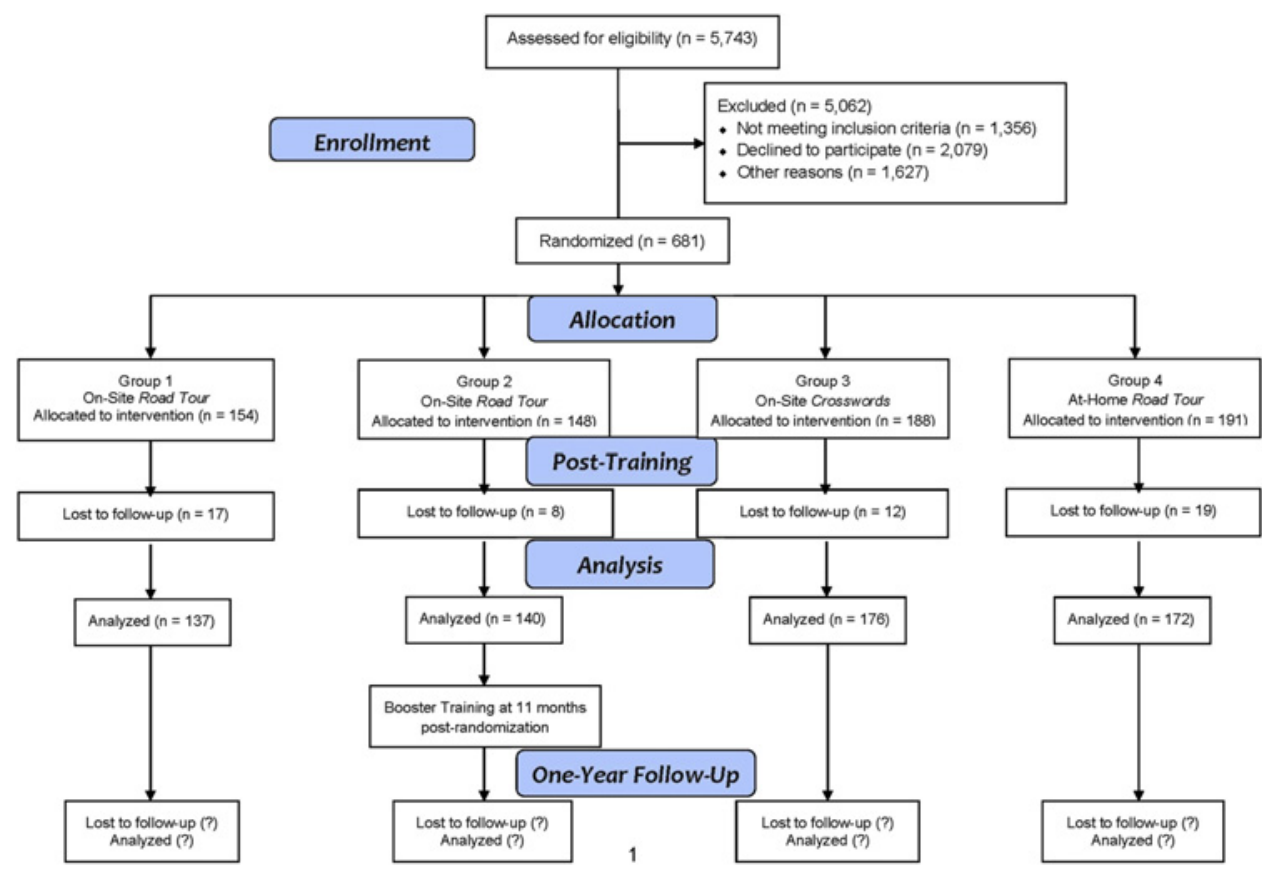

unable to reach 1627. Upon achieving telephone contact with the remainder, 2079 declined to participate. We then conducted brief screening interviews among interested patients. These screening interviews identified potential participants who met any of the exclusion criteria that could not be ascertained using Epic: (1) significant cognitive impairment evidenced by $\geq 3$ errors on a 10 -item mental status exam, ${ }^{38}$ (2) self-reported uncorrected visual acuity problems that would interfere with using a PC, (3) not having a PC with a CD-ROM in the home, (4) not having internet access and (5) having previously used a cognitive training programme. This led to the exclusion of 1356 potential participants.

\section{Informed consent and baseline interviews}

After successfully completing the screening telephone interview, eligible patients were scheduled for a $2 \mathrm{~h}$ appointment in our laboratory where the purpose and design of the study were explained, and written informed consent was obtained. The informed consent process took about $15 \mathrm{~min}$. After providing informed consent, the 681 enrollees were administered their baseline (randomisation) interviews by trained research assistants using computer-assisted interviewing protocols. The baseline interviews took about $1.5 \mathrm{~h}$ and included the neuropsychological assessments, health outcomes and covariates described below. Immediately after their baseline interview, participants were randomised to one of the four study groups.

\section{Randomisation procedure}

The study biostatistician (MPJ) determined the order of assignments using a computer-generated list of random numbers and a 3:3:4:4 ratio based on a priori power calculations. Block randomisation was used to maintain balance on two age-strata $(50-64$ and $\geq 65)$. Block sizes 
of 4,8 and 12 were randomly varied. The assignment for each participant's ID number was recorded on a participant letter and then sealed in an opaque envelope with only the ID number visible. Two age-strata specific boxes containing the assignment envelopes were stored in a locked cabinet in the Project Coordinator's office. The Project Coordinator (MMD) had the responsibility of unsealing the envelope (from the appropriate agestratum box) and revealing each participant's group assignment.

\section{Cognitive processing speed outcomes}

The six IHAMS neuropsychological assessments are: (1) the UFOV PC mouse version ${ }^{19}$; (2) the Symbol Digit Modalities Test (SDMT) ${ }^{39}$; (3) the Trail Making A and B Tests (TMT) ${ }^{40}$; $(4)$ the Controlled Oral Word Association Test (COWAT) ${ }^{41}$; (5) the Digit Vigilance Test $(\mathrm{DVT})^{42}$; and (6) the Stroop Colour and Word Test (Stroop). ${ }^{43}$ The UFOV is the primary outcome and was administered at randomisation and post-training (6-8 weeks postbaseline), and will be readministered at 1 year postrandomisation. The UFOV includes three subtests-stimulus identification, divided attention and selective attention-each scored from $17-500 \mathrm{~ms}$ reflecting the shortest exposure time at which the participant could correctly perform each subtest $75 \%$ of the time, with a composite milliseconds outcome score ranging from 51-1500 ms.

The SDMT, TMT, COWAT, DVT and Stroop tests are secondary outcome measures, were all administered at randomisation and will be readministered at 1 year postrandomisation. SDMT captures divided attention and processing speed, and is based on how many of 110 possible digit-symbol pairs were scored as correct pairs by the participant in $90 \mathrm{~s}$. TMT assesses visual scanning ability, processing speed and set-shifting/executive functioning, and is coded as the number of seconds needed to correctly complete connection of the number and number-letter sets. COWAT assesses verbal fluency based on the number of unique words beginning with the letter $\mathrm{C}$ (or $\mathrm{F}$ or $\mathrm{L}$ ) generated by the participant during $60 \mathrm{~s}$, with a composite score of the number of correct words used across the three letter trials. DVT assesses sustained attention and psychomotor speed, is performed by crossing out randomly placed $6 s$ in 59 rows of numbers and is scored as the error and time totals. The Stroop assesses processing speed and executive functioning, and is scored as the correct number of words, colours and colour-words identified in $45 \mathrm{~s}$ on each subtest.

\section{Health outcomes}

Three health outcome measures-HRQoL, depressive symptoms and sense of control-are included. Each has established reliability and validity, and yet is relatively brief. The 36 -item SF-36 ${ }^{44}$ is used to measure HRQoL. Scores on each of its eight subscales are transformed to range from 0 (worst health) to 100 (best health).
Depressive symptoms are measured using the 12-item Center for Epidemiologic Studies Depression scale (CESD-12). ${ }^{45}$ CESD-12 scores range from 0 (no symptoms acknowledged) to 36 (all 12 symptoms acknowledged to occur most or all of the time), with scores $\geq 9$ being the screening threshold for suspected clinical depression. ${ }^{34}$ Sense of control is measured using Mirowsky and Ross' eight-item $2 \times 2$ Index. $^{46}$ The sense of control score is the sum of the item responses and ranges from maximally denying $(-16)$ to maximally claiming control $(+16)$.

\section{Covariates}

To adjust for potential heterogeneity across the treatment groups, several covariates were obtained at randomisation. These included various sociodemographic characteristics, multiple indicators of socioeconomic status, medical history, ADLs and IADLs, perceived stress, self-efficacy and, attitudes towards and the use of computers in everyday life, among others.

\section{Road Tour training programme}

In November 2007, Posit Science Corporation (San Francisco, California) acquired the rights to Ball and Roenker's original visual speed-of-processing training programme. ${ }^{19} 20{ }^{26}$ While Posit Science retained all of the original tasks, it modified the delivery platform to be user-friendly and self-administered, improving the ease of dissemination and implementation. The addition of certain game elements also improves user engagement and enhances compliance. The resulting second-generation computerised speed of processing training programme is known as Road Tour and is commercially available (http://www.positscience.com/our-products).

Road Tour's appearance is shown in figure 2. After clicking on the start button to initiate training, figure $2 \mathrm{~B}$ is shown. Here, both the licence plate area and the eight circular locations in the near orbit surrounding it are empty. The empty licence plate is then replaced, as in figure $2 \mathrm{C}$, with the target vehicle, either a car or a truck. Similarly, the eight empty circular locations surrounding the licence plate are then replaced with seven distracter stimuli (rabbit crossing signs) or the target sign (Route 66 ). The stimuli (car vs truck and rabbit crossing vs Route 66 sign) are presented for a specified time and are then replaced by figure 2D. The amount of time that figure $2 \mathrm{C}$ remains on the screen before being replaced by figure 2D is measured in milliseconds. In figure 2E, both target vehicles (the car and truck) are presented in the centre of the screen. The user first clicks on the correct target vehicle (car or truck) and then on the circular location where the correct peripheral target (Route 66 sign) appeared (figure 2F). The goal is to improve cognitive processing speed by progressively reducing the milliseconds of exposure that figure 2C remains on the screen with subsequent correct identification of both the stimuli (car or truck) and target (Route 66) sign. 

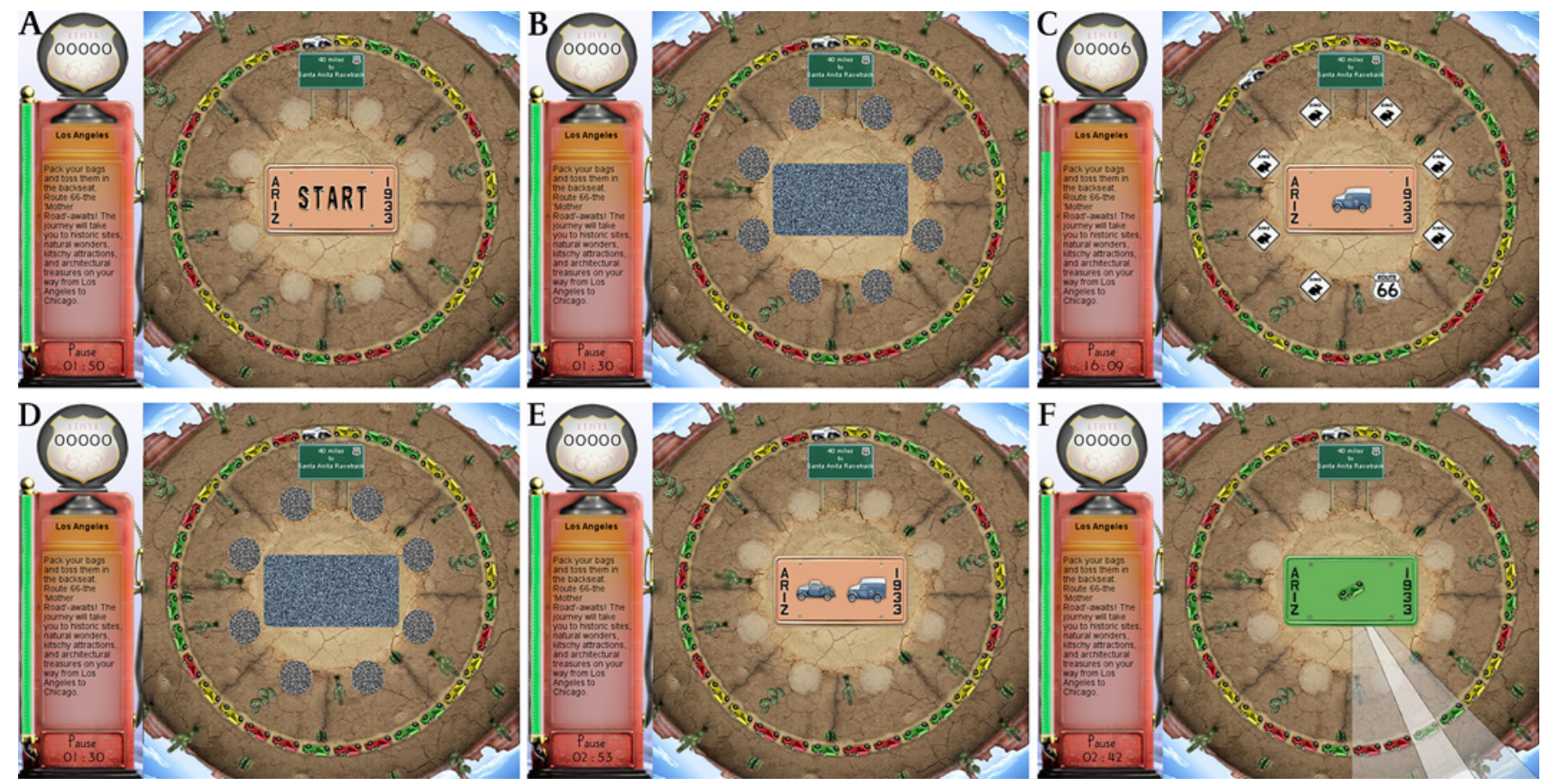

Figure 2 The initial Road Tour sequence

The training programme is tailored to the participant's performance such that it maintains a $75 \%$ success rate before advancing to a shorter exposure time. The gaming aspect involves the user deciding where to place a car in the ring of cars when a trial (identification of the vehicle and Route 66 sign) is correct (figure $2 \mathrm{~F}$ ). When car placement produces a sequence of three cars of the same colour, these cars disappear, and the user's car moves around the ring. This gaming element increases the user's engagement in the exercise. Each lap around the ring gets the user's car closer to the next destination. In the initial (early) trials of the exercise, there are only seven distracters within the near orbit around the licence plate. As the user progresses, three changes occur to increase task difficulty: (a) the target visual field expands (figure $3 \mathrm{~A}-\mathrm{C}$ ) by progressing outward from the licence plate to add medium and distal orbits; (b) these are accompanied by an increasing number of distracters to populate all three orbits (up to 47; figure 4A,B); and (c) the vehicle pairs morph through nine different stages or pairs to become more similar and thus more difficult to differentiate (figure 5).

Road Tour captures the participant's experience in two ways, both of which are routinely sent in the background to Posit Science and our research laboratory over the internet using secure file encryption protocols. One assessment is the amount of time spent playing Road Tour. The targeted standard training dose was $10 \mathrm{~h}$. Use can also be assessed by the percentage of completion of all 81 of the available exercise sets. In addition to monitoring use, Road Tour also administers an assessment of visual-processing speed at random intervals. This assessment is the number of milliseconds that figure $2 \mathrm{C}$ must remain exposed to the participant to achieve the specified correct identification (success) rate. It is estimated as the log mean of two randomly interleaved zippy estimation by sequential testing ${ }^{47}$ adaptive Bayesian algorithms ${ }^{48}$ which employ a cumulative Gaussian curve starting at $6.75 \%$ (chance correct rate) and ending at 95\% (100\% minus 5\% lapse rate) with the threshold set at $50 \%$.

\section{Group 1}

Immediately after completing their informed consent and baseline interviews, participants randomly assigned to Group 1 (on-site Road Tour training without boosters) were scheduled for their first $2 \mathrm{~h}$ session in our laboratory, which includes two identical training rooms configured with five private PC workstation areas. The Road Tour training software is on the PCs in one training room, and the computerised crossword puzzle software is on the PCs in the other. At their first session, Group 1 participants were assigned their studyspecific ID number and, after receiving about 5-10 min of scripted instruction on how to use Road Tour, were asked to use the training programme for the remainder of that session. Participants were then scheduled for their next $2 \mathrm{~h}$ training session, which usually occurred the following week. A total of five, weekly $2 \mathrm{~h}$ training sessions were scheduled for the standard training dose. After completing $10 \mathrm{~h}$ of training, or by $6-8$ weeks postrandomisation, whichever came first, Group 1 participants were invited back to the laboratory for their post-training assessments using the UFOV test.

\section{Group 2}

Participants randomly assigned to Group 2 (on-site Road Tour training with boosters) were treated the same as 

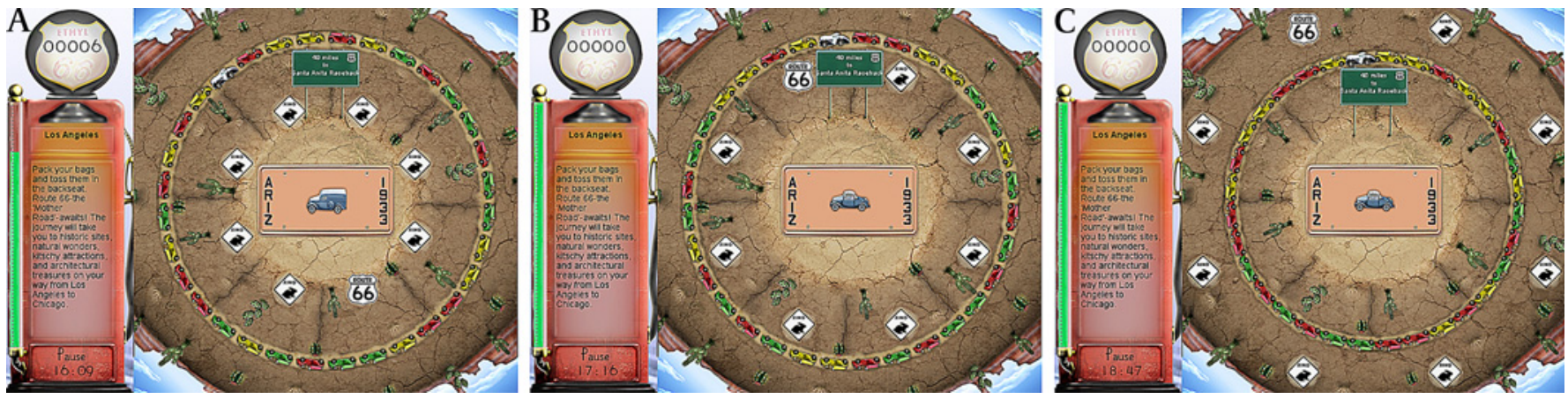

Figure 3 Expansion of the target visual field

those in Group 1 with one exception. Unlike Group 1 participants, Group 2 participants were invited back to our laboratory for two $2 \mathrm{~h}$ booster training sessions at 11 months postrandomisation. Group 2 participants also completed additional UFOV testing both before and after their booster training.

\section{Group 3}

Participants randomised to Group 3 (on-site computerised crossword puzzle training; attention control) were treated the same as participants randomised to Group 1, with one exception. Instead of using Road Tour, Group 3 participants were taken to our second training room and instructed on how to use the computerised crossword puzzle programme (Boatload of Crosswords, Boatload Puzzles, LLC, Yorktown Heights, New York).

\section{Group 4}

Participants randomly assigned to Group 4 (at-home Road Tour training) were scheduled for their first session in our laboratory immediately after completing their informed consent and baseline interviews. They, however, were taken to a third room in which they were assigned their study-specific ID number and were then shown (step by step) how to load the software on a PC. After this, they received about 5-10 min of scripted instruction on how to use Road Tour and then practised using it for about 10-15 min. Group 4 participants were then sent home with the CD containing the Road Tour software to load on their home PCs, as well as a detailed set of step-by-step instructions containing all of the screenshots that they would encounter in doing so. They were also given the phone number and email informa- tion for contacting the Project Coordinator (MMD) to answer any questions they might have about loading the software onto their home PCs. Group 4 participants were asked to use Road Tour at home for $10 \mathrm{~h}$ or more during the next 5-6 weeks. At 6-8 weeks postrandomisation, Group 4 participants were invited back to the laboratory for their post-training assessments using the UFOV test. At that time, they were reminded that they could continue using Road Tour as often as they liked.

\section{Analysis}

For the purposes of statistical modelling, we define three mutually exclusive $1-0$ binary indicators G1, G2 and G4 to indicate whether the participant is in the on-site speed of processing intervention without boosters, the on-site speed of processing intervention with boosters, or the athome speed of processing group. The on-site crossword puzzle (attention control; G3) group participant will have all three of these indicators set to zero. Other covariates are contained in the vector $X$. For continuous outcomes such as visual processing speed we will use multiple linear regression models $^{49}$ that may be expressed in their simplest form as:

$$
\mathrm{Y}_{12}=\beta_{0}+\beta_{1} \mathrm{Y}_{\mathrm{b}}+\beta_{2} \mathrm{G} 1+\beta_{3} \mathrm{G} 2+\beta_{4} \mathrm{G} 4+\beta_{5} \mathrm{X}+\varepsilon
$$

where $\mathrm{Y}_{12}$ is the dependent variable at the 12-month postrandomisation assessment, $\beta_{\mathrm{o}}$ is the intercept, $\beta_{1}$ is the coefficient for $Y_{b}$ (the baseline (randomisation) value of the dependent variable), $\beta_{2}$ is the coefficient for G1 (Group 1), $\beta_{3}$ is the coefficient for G2 (Group 2), $\beta_{4}$ is the coefficient for G4 (Group 4), $\beta_{5}$ is the vector of
Figure 4 Increasing the number of visual distractors
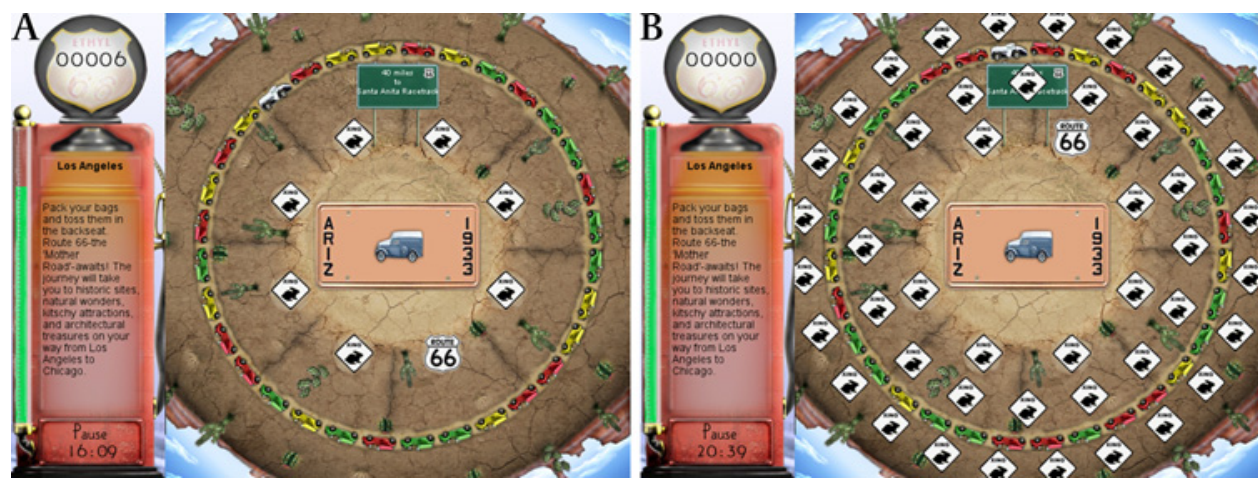

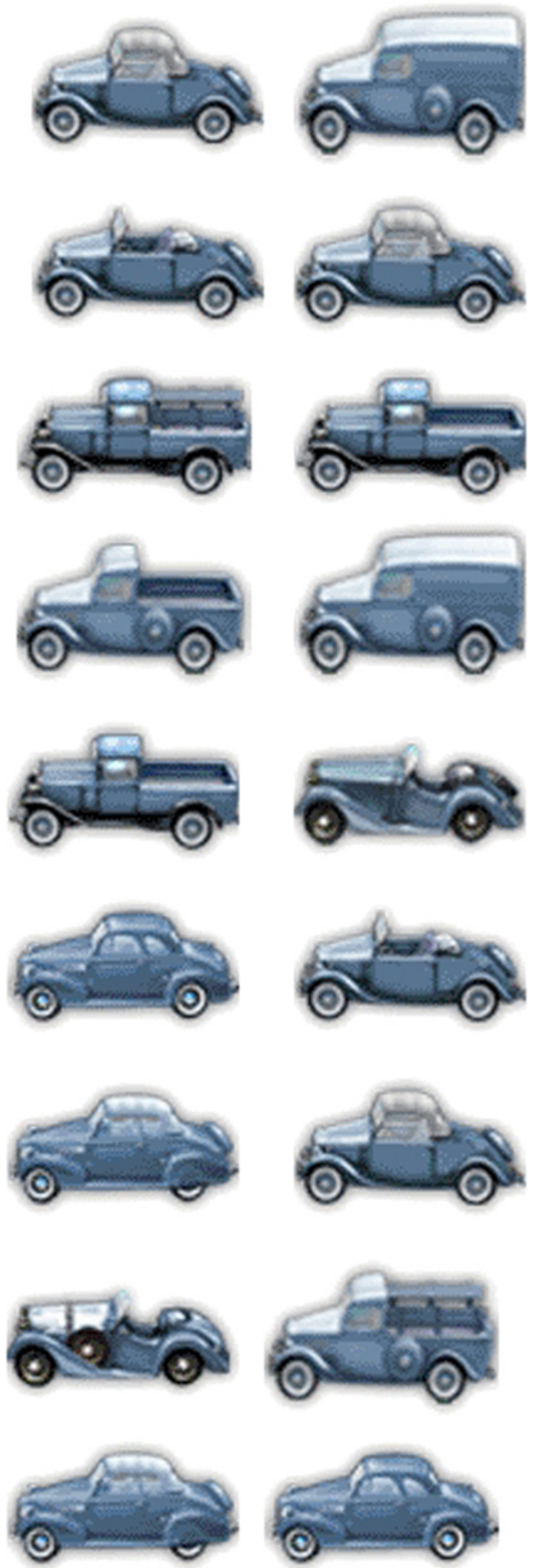

Figure 5 Vehicle morphing

coefficients for $\mathrm{X}$ (the vector of covariates), and $\epsilon$ is the error term. The effect of $\beta_{1}$ represents a stability coefficient; the effects of $\beta_{2}, \beta_{3}$ and $\beta_{4}$ represent the effects of being randomised to the three Road Tour intervention groups (Groups 1, 2 and 4), respectively, on changes in the dependent variable compared with those observed for the attention control group (Group 3); and the effects of $\beta_{5}$ represent the effects of the covariates on changes in the dependent variable. When the dependent variable is a count measure, we will use Poisson or negative binomial regression techniques, and for binary outcomes (such as the onset of suspected clinical depression (CESD-12 scores $\geq 9$ )), we will use logistic regression.

\section{Modifications to the original protocol}

The protocol entry for IHAMS that was originally submitted to http://www.ClinicalTrials.gov was subsequently modified to reflect two changes. First, our original power calculations in the USA NIH Challenge Grant Application were based on a meta-analysis of previously published studies using Ball and Roenker's intervention. Once IHAMS was funded, we used original data from the ACTIVE baseline and 1-year follow-up interviews to estimate statistical power and sample size requirements more accurately. Those analyses indicated that a total of 600 participants across the four study groups would yield at least $80 \%$ power to detect an effect size 0.25 at $\alpha=0.05$ with a two-sided test. Therefore, we changed the sample size goals from 900 to 600 , and ultimately enrolled and randomised 681 participants. Second, all six neuropsychological tests were originally listed in the protocol as primary outcomes. Because only the UFOV was used in ACTIVE, however, we could only power the study based on it. Therefore, the five other neuropsychological tests were changed to secondary outcomes.

\section{ETHICS AND DISSEMINATION}

IHAMS was viewed as a minimal risk trial by the University of Iowa IRB for three reasons. First, no adverse effects were reported from the earlier version that was used in ACTIVE. Second, Boatload of Crosswords, the computerised crossword puzzle program, is the most popular puzzle game commercially available. Third, our IRB protocol (200908789) established procedures to ensure that participation was voluntary, that participants could quit at any time they chose, that signed informed consent was obtained, that confidentiality was maintained and that data security was rigorous. Participants received $\$ 25$ for completing their randomisation interviews, $\$ 5$ for each UFOV test and $\$ 50$ for completing the 1 -year postrandomisation interview.

We will disseminate the results from IHAMS via conference presentations and journal publications. Four subsequent journal articles will focus on (1) the posttraining UFOV results, (2) the 1-year postrandomisation results on all six neuropsychological assessments, (3) the health outcome results at 1-year postrandomisation and (4) effectiveness-derived dose-response curves. If the 1-year postrandomisation results demonstrate the efficacy and effectiveness of Road Tour, Posit Science will work with government agencies to make the programme available for wide-scale implementation 'at only a fraction of the current per-user cost.'

Author affiliations:

${ }^{1}$ Department of Health Management and Policy, University of lowa, Iowa City, Iowa, USA 
${ }^{2}$ Department of Medicine, University of lowa, lowa City, lowa, USA

${ }^{3}$ Department of Nursing, University of lowa, lowa City, lowa, USA

${ }^{4}$ Department of Psychology, University of lowa, lowa City, Iowa, USA

${ }^{5}$ Iowa City VA Health Care System, Iowa City, lowa, USA

${ }^{6}$ Department of Biostatistics, University of lowa, lowa City, lowa, USA

${ }^{7}$ Department of Neurology, University of Utah, Salt Lake City, Utah, USA

Acknowledgements The authors acknowledge and applaud the 681 participants from the University of lowa FCC general internal and family medicine clinics. Without their participation and support, this study would not have been possible. The authors also acknowledge the research assistants, work-study students and other support staff involved in IHAMS.

Correction notice The "To cite: ..." information and running footer in this article have been updated with the correct volume number (volume 1).

Funding This study was supported by US NIH grant RC1 AG-035546 to FDW.

Competing interests The Road Tour computerised visual speed of processing intervention used in lowa Healthy and Active Minds Study (IHAMS) is commercially available from Posit Science Corporation (San Francisco, California). None of the members of the investigative team have any conflicts of interest or conflicts of commitment involving Posit Science. Specifically, no one on the investigative team will financially profit in any way from the use of Road Tour in IHAMS, or in any subsequent study using Road Tour. Posit Science acquired ownership in October 2007 of the original speed of processing intervention that was used in the multisite ACTIVE RCT on which FDW was an original co-investigator (at the ACTIVE Indiana University site). The ACTIVE speed of processing intervention had been developed earlier by $\mathrm{K}$ Ball (ACTIVE site-PI at the University of Alabama-Birmingham) and D Roenker (at Western Kentucky University). In collaboration with $\mathrm{K}$ Ball and D Roenker, Posit Science subsequently developed the second-generation, value-added version of the visual speed of processing intervention known as Road Tour and used here in IHAMS. From December 2007 to March 2009, FDW had a limited, part-time consulting arrangement ( 15 days, total) with Posit Science to support additional analyses of the first 5 years of the ACTIVE follow-up data that had not been identified in the original ACTIVE protocols or funded by the various US NIH grants supporting ACTIVE. This arrangement was approved in advance by the ACTIVE Executive Committee (which included the US NIH project officers), and was sanctioned by the Provost of the University of lowa. After terminating this limited, part-time consulting arrangement with Posit Science, FDW applied in April 2009 for, and was awarded in September 2009 the US NIH Challenge Grant known as IHAMS. Posit Science provided the 700 copies of Road Tour used in IHAMS at no cost whatsoever. Furthermore, in its letter of commitment to IHAMS and the US NIH, Posit Science stated should the results support the efficacy and effectiveness of Road Tour, they will 'work with agencies at the federal government to make the programme available for wide-scale implementation at only a fraction of the current per-user cost.'

Ethics approval Ethics approval was provided by the University of lowa Institutional Review Board (IRB-03 protocol 200908789).

Contributors FDW is the principal investigator on the study, wrote the original proposal, supervised the trial, conducted all of the analyses and drafted the manuscript. MWVW is co-principal investigator on the study, collaborated on the original proposal, co-supervised the trial and reviewed the analyses reported here as well as the manuscript itself. MBH is a postdoctoral fellow working on the study, trained all of the interviewers, supervised the scoring of the neuropsychological tests and reviewed the manuscript. MPJ is the study biostatistician, devised the randomisation protocol, reviewed all of the analyses and reviewed the manuscript. RM is a co-investigator on the study, reviewed all of the ethics, consent and IRB documents, and reviewed the manuscript. TML was a study research assistant who assisted with piloting the interview protocol, conducted randomisation interviews and reviewed the manuscript. KD is the study neuropsychologist, supervised selection of the neuropsychological tests, reviewed the psychometric analyses and reviewed the manuscript. CG is the medical director of the FCC General Medicine Clinic, participated in subject recruitment and reviewed the manuscript. SW is the medical director of the FCC Family Medicine Clinic, participated in subject recruitment and reviewed the manuscript. MMD is the project coordinator.

Provenance and peer review Not commissioned; peer reviewed for ethical and funding approval prior to submission. IHAMS was not a commissioned study.
It was externally peer reviewed by a panel of Distinguished Editors (ZRG1 RPHB-E 58) convened by the US NIH to select approximately 200 Challenge Grant Applications (RC1s) submitted in response to the American Recovery and Reinvestment Act of 2009 (from a pool of about 23000 submitted proposals).

Data sharing statement No later than 3 years after the collection of the 1-year postrandomisation interviews, we will deliver a completely deidentified data set to an appropriate data archive for sharing purposes.

\section{REFERENCES}

1. Birren JE, Woods AM, Williams MV. Behavioral slowing with age: causes, organization, and consequences of slowing. In: Poon LW, ed, Aging in the 1980s: Psychological Issues. Washington, DC: American Psychological Association, 1980:293-308.

2. Edwards JD, Wadley VG, Myers RS, et al. Transfer of a speed of processing intervention to near and far cognitive functions. Gerontology 2002;48:329-40.

3. Madden DJ. Four to ten milliseconds per year: age-related slowing of visual word identification. J Gerontol 1992;47:P59-68.

4. Roenker DL, Cissell GM, Ball KK, et al. Speed-of-processing and driving simulator training result in improved driving performance. Hum Factors 2003;45:218-33.

5. Cronin-Golomb A. Color vision, object recognition, and spatial localization in aging and Alzheimer's disease. In: Hof PR, Mobbs CV eds. Functional Neurobiology of Aging. San Diego, CA: Academic Press, 2001:517-30.

6. McDowd JM, Shaw RJ. Attention and aging: a functional perspective. In: Craik FIM, Salthouse TA, eds. The Handbook of Aging and Cognition. Mahwah, NJ: Lawrence Erlbaum Associates, 2000:221-92.

7. Schneider BA, Pichora-Fuller MK. Implications of perceptual deterioration for cognitive aging research. In: Craik FIM, Salthouse TA, eds. The Handbook of Aging and Cognition. Mahwah, NJ: Lawrence Erlbaum Associates, 2000:155-220.

8. Albert MS, DeKosky ST, Dickson D, et al. The diagnosis of mild cognitive impairment due to Alzheimer's disease: Recommendations from the National Institute on Aging and Alzheimer's Association workgroup. Alzheimers Dement 2011;7:270-9.

9. Jack CR, Albert M, Knopman DS, et al. Introduction to revised criteria for the diagnosis of Alzheimer's disease: National Institute on Aging and the Alzheimer's Association workgroup. Alzheimers Dement 2011;7:257-62.

10. McKhann GM, Knopman DS, Chertkow D, et al. The diagnosis of dementia due to Alzheimer's disease: recommendations from the National Institute on Aging and the Alzheimer's Association workgroup. Alzheimers Dement 2011;7:263-9.

11. Sperling RA, Aisen PS, Beckett LA, et al. Toward defining the preclinical stages of Alzheimer's disease: recommendations from the National Institute on Aging and the Alzheimer's Association workgroup. Alzheimers Dement 2011;7:280-92.

12. Burdick DJ, Rosenblatt A, Samus QM, et al. Predictors of functional impairment in residents of assisted living facilities: the Maryland Assisted Living Study. J Gerontol A Biol Sci Med Sci 2005;60A:258-64.

13. Cahn-Weiner DA, Malloy PF, Boyle PA, et al. Prediction of functional status from neuropsychological tests in community-dwelling elderly individuals. Clin Neuropsychol 2000;14:187-95.

14. Hertzog C, Kramer AF, Wilson RS, Lindenberger U. Enrichment effects on adult cognitive development: can the functional capacity of older adults be preserved and enhanced? Psychol Sci Pub Interest 2008;9:1-65.

15. Salthouse TA. Consequences of age-related cognitive declines. Ann Rev Psychol 2012;63:In press.

16. Salthouse TA. The processing-speed theory of adult age differences in cognition. Psychol Rev 1996;103:403-28.

17. Schaie KW. Intellectual Development in Adulthood: The Seattle Longitudinal Study. New York: Cambridge University Press, 1996.

18. Smith AD, Earles JLK. Memory changes in normal aging. In: Blanchard-Fields F, Hess TM, eds. Perspectives on Cognitive Change in Adulthood and Aging. New York: McGraw-Hill, 1996:192-220.

19. Edwards JD, Vance DE, Wadley VG, et al. Reliability and validity of Useful Field of View test scores as administered by personal computer. J Clin Exp Neuropsychol 2005;27:529-43.

20. Wadley VG, Benz RL, Ball KK, et al. Development and evaluation of home-based speed of processing training for older adults. Arch Phys Med Rehabil 2006;87:757-63.

21. Clay OJ, Edwards JD, Ross LA, et al. Visual function and cognitive speed of processing mediate age-related decline in memory span and fluid intelligence. J Aging Health 2009;21:547-66. 
22. Lemke U, Zimprich D. Longitudinal changes in memory performance and processing speed in old age. Aging Neuropsychol Cognit 2005;12:57-77.

23. Zimprich DM. Can longitudinal changes in processing speed explain longitudinal changes in fluid intelligence? Psychol Aging 2002; 17:690-5.

24. Rosnick CB, Small BJ, Graves AB, et al. The association between health and cognitive performance in a population-based study of older adults: the Charlotte County Healthy Aging Study (CCHAS). Aging Neuropsychol Cognit 2004;11:89-99.

25. Salthouse TA. When does age-related cognitive decline begin? Neurobiol Aging 2009;30:507-14.

26. Ball KK, Edwards JD, Ross LA. The impact of speed of processing training on cognitive and everyday functions. J Gerontol B Psychol Sci Soc Sci 2007;62B:19-31.

27. Jobe JB, Smith DM, Ball KK, et al. ACTIVE: a cognitive intervention trial to promote independence in older adults. Controlled Clin Trials 2001;22:453-79.

28. Ball KK, Berch DB, Helmers KF, et al. Effects of cognitive training interventions with older adults: a randomized controlled trial. JAMA 2002;288:2271-81

29. Willis SL, Tennstedt SL, Marsiske M, et al. Long-term effects of cognitive training on everyday functional outcomes in older adults. JAMA 2006;296:2805-14.

30. Wolinsky FD, Mahncke HW, Kosinksi M, et al. The ACTIVE cognitive training trial and predicted medical expenditures. BMC Health Serv Res 2009;9:109.

31. Wolinsky FD, Unverzagt FW, Smith DM, et al. The effects of the ACTIVE cognitive training interventions on clinically relevant declines in health-related quality of life. J Gerontol B Psychol Sci Soc Sci 2006;61B:S281-7.

32. Wolinsky FD, Unverzagt FW, Smith DM, et al. The ACTIVE cognitive training trial and health-related quality of life: protection that lasts for five years. J Gerontol A Biol Sci Med Sci 2006;61A:1324-9.

33. Wolinsky FD, Vander Weg MW, Martin R, et al. The effect of speed of processing training on depressive symptoms in ACTIVE. J Gerontol A Biol Sci Med Sci 2009;64A:468-72.

34. Wolinsky FD, Mahncke HW, Vander Weg MW, et al. The ACTIVE cognitive training interventions and the onset of and recovery from suspected clinical depression. J Gerontol B Psychol Sci Soc Sci 2009;64B:577-85.
35. Wolinsky FD, Mahncke HW, Vander Weg MW, et al. Speed of processing training improves self-rated health in older adults: enduring effects observed in the multi-site ACTIVE study. Int Psychogeriatr 2010;22:470-8.

36. Wolinsky FD, Vander Weg MW, Martin R, et al. Cognitive training improves internal locus of control among older adults. J Gerontol B Psychol Sci Soc Sci 2010;65B:470-8.

37. Ball KK, Beard BL, Roenker DL, et al. Age and visual search: expanding the useful field of view. J Opt Soc Am A 1988;5:2210-19.

38. Pfeiffer EA. A short portable mental status questionnaire for the assessment of organic brain deficit in elderly patients. J Am Geriatr Soc 1975;23:433-41.

39. Smith A. Symbol Digit Modality Test. Los Angeles, CA: Western Psychological Services, 1982.

40. Reitan RM, Wolfson D. The Halstead-Reitan Neuropsychological Test Battery: Therapy and Clinical Interpretation. Tucson, AZ: Neuropsychological Press, 1985

41. Benton AL, Hamsher K, Silvan AB. Multilingual Aphasia Examination 3rd edn. Iowa City, IA: AJA Associates, 1994

42. Lewis R, Rennick PM. Manual for the Repeatable CognitivePerceptual-Motor Battery. Gross Point, MI: Axon, 1979.

43. Golden CJ. The Stroop Color and Word Test. Chicago: Stoelting Company, 1978.

44. McHorney CA, Ware JE, Raczek R. The MOS 36-Item Short-Form health survey (SF-36): II. Psychometric and clinical tests of validity in measuring physical and mental health constructs. Med Care 1993;31:247-63.

45. Kohout FJ, Berkman LF, Evans DA, et al. Two shorter-forms of the CESD depression symptoms index. J Aging Health 1993;5:179-93

46. Ross C, Mirowsky J. Eliminating defense and agreement bias from measures of the sense of control: a $2 \times 2$ Index. Soc Psychol Qtly 1991;54:127-45.

47. King-Smith PE, Grigsby SS, Vingrys AJ, et al. Efficient and unbiased modifications of the QUEST threshold method: theory, simulations, experimental evaluation and practical implementation. Vision Res 1994;34:885-912.

48. Watson AB, Pelli DG. QUEST: a Bayesian adaptive psychophysical method. Percept Psychophysics 1983;33:113-20.

49. Kessler RC, Greenberg DF. Linear Panel Analysis: Models of Quantitative Change. New York: Academic Press, 1981. 